\title{
A DISCUSSION OF PROBLEMS CONCERNING PULPLESS TEETH AND PULP DEVITALIZATION
}

\author{
Authentic Information and Representative Opintons, Compiled \\ From Responses to a QUESTIONNAIRE ${ }^{1}$ \\ (Fourth Part) $)^{2}$
}

ELMER S. BEST

Minneapolis, Minnesola

CONTENTS

I. Introduction. .......................................... 384

II. Copies of the questions in the questionnaire; with the author's summary, under each, of his conclusions from the facts and opinions respectively stated by the correspondents $\ldots \ldots \ldots \ldots \ldots \ldots \ldots \ldots \ldots \ldots \ldots \ldots \ldots \ldots \ldots \ldots \ldots, 384$

Questions in the first part (pp. 1-102 of this volume):

(I) In the treatment of vital deciduous teeth, where the decay is so extensive that its entire removal would mean the exposure of the pulp, what do you advise and what is your procedure? Also, how do you proceed where the pulp is already exposed?.............. 384

(II) In the treatment of deciduous teeth where the pulps have become nonvital and infected, what is your procedure and what do you advise? 384

(III) In case the root ends of permanent teeth are not completely formed and the pulp has decomposed, what is your procedure and what do you advise?.................................. 385

(IV) In case the root ends of permanent teeth are not completely formed, and the pulp is still vital but exposed, as a result of decay or trauma, what is your procedure and what do you advise?............ 385

(V) In case the vital pulp is covered with a thin layer of decalcified dentine, what is your procedure and what do you advise?...... 385

(VI) At what age do you consider it good practice to begin bridgework that necessitates cutting into vital teeth? Do you consider that the location in the mouth is a governing factor? .............. 385

1 Best: Journal of Dental Research, 1919, i, p. 523; 1920, ii, p. 285; 1922, iv, pp. 1, 129, and 225.

2 The first part was published in the Journal of Dental Research, 1922, iv, p. 1 (March); the second part, p. 129 (June); the third part, p. 225 (September). The four parts of which this compilation consists will be issued in book form on or about June 1, 1923. 
(VII) When permanent teeth of young patients are extracted on account of apical infection, do you retain the spaces with orthodontic appliances, use bridgework, or pay no attention to the spaces created

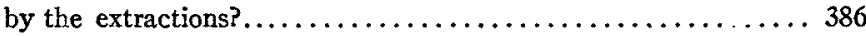

(VIII) Where deciduous teeth are extracted, do you, under any circumstances, advise retention of the spaces, with orthodontic appliances, for the eruption of the permanent teeth?................ 386

(IX) What were the conditions that caused the death or removal of so many pulps in the past? To what extent are these conditions now under control and pulp removal unnecessary?............. 386

(X) What are the causes of pulp death over which we still have no control? 386

(XI) In a case where decay is in actual contact with a vital pulp, do you advocate (a) removal of all decay and attempted pulp capping; or (b) removal of the decay followed by pulp removal, (c) leaving just enough decalcified dentine to prevent actual exposure and giving the case a treatment? $\left(c^{\prime}\right)$ What does such treatment consist of? How long have you practised it? Do you consider it a good procedure to follow? ......................... 387

(XII) How early do you begin oral prophylaxis for your patients?....... 387

(XIII) Outline your plans for preventing decay and pulp death in the teeth of young patients............................... 387

(XIV) Is it your habit to give the mouths of your patients prophylactic supervision during the time they are in your care? If so, how frequently do you see the patients for this work?........... 388

(XV) In deciding why the radiograms of some non-vital teeth show areas indicative of apical disease, and some do not, and also why some persons with such teeth display symptoms suggestive of focal infection while others do not, is it probable that different factors contribute to the two conditions at the ends of the roots? (Be sure to mention whether or not you believe the development of apical infection is solely a matter of diminished resistance on the part of the patient.).................................. 388

(XVI) In the case of a non-vital tooth showing apical infection, outline methods by which such infection can be removed and the tooth retained. Do you consider that your procedure gives permanently satisfactory results? ............................ 388

(XVII) Approximately what number of non-vital non-infected teeth would you advise leaving in any one mouth (a) in the absence of symptoms of focal infection; (b) in the presence of symptoms of focal infection? Do you make any distinction, in this regard, as far as age of the patient is concerned? .............................

Questions in the second part (pp. 129-212 of this volume):

(XVIII) In a case showing symptoms of focal infection, to what extent would you go in trying to save a non-vital tooth that apparently had no apical infection, provided such a tooth was a valuable factor in the reconstruction of the mouth? $\ldots \ldots \ldots \ldots \ldots \ldots \ldots \ldots .38$ 
(XIX) In case a radiogram shows a tooth with a pulp chamber and portions of one or more roots only partly filled, how do you determine whether the unfilled apical portions of the roots contain vital pulp-tissue, or non-vital tissue, or no pulp-tissue at all-all three conditions being supposedly free from apical infection? ... 389

(XX) In the reconstruction of a mouth, do you believe in removing nonvital teeth that do not show, radiographically, pathological conditions at the apices?............................ 390

(XXI) Do you believe in root resection as a permanent operation? On which teeth? Do you feel that, in such an operation, the pulpcanal and dentine should receive special attention? Of what should such trea tment consist?...................... 390

(XXII) Should a tooth that is abscessed be extracted during the active process of inflammation, or is such an operation as a routine procedure contraindicated?........................... 391

(XXIII) For the extraction of a considerable number of teeth in a case of serious infection, how do you proceed? Do you remove a few at a time or all at one operation? ..................... 391

(XXIV) Do you consider that unerupted and impacted teeth should be removed, regardless of their location, when they cannot be brought into their proper places in the arch?................ 391

(XXV) Under what conditions, and in what locations, in the mouth, is it justifiable to remove a decomposed pulp and to fill the canal or canals? Give a list of such conditions................. 391

(XXVI) Under what conditions, and in what locations in the mouth, is it justifiable to remove a vital pulp and to fill the root canals with an artificial substitute? Give a list of such conditions........ 391

(XXVII) When a tooth, to be extracted, has an apical infection, should the infected region be curetted after the extraction, or should it be left undisturbed? Would your decision to curette be the same if the granuloma came out with the root?

(XXVIII) Upon what basis do you decide what to do with the various teeth in a mouth that contains a great number of non-vital teeth; some showing apical involvement and some not; some with partially and others with completely filled roots; some with exposed vital pulps and some with dead pulps? Go sufficiently into detail so that your answer may serve as a guide in general practice....... 392

(XXIX) In the case of a maxillary-sinus infection that did not respond to treatment, what disposition would you make of any non-vital molars or bicuspids on the side affected? ................ 392

(XXX) In the reconstruction of a mouth, would you advise the removal of one or more vital pulps, if that procedure enabled you to use a more satisfactory type of bridge than would be possible if such pulps were not removed? If you ever advise such a procedure, does the patient's age and condition influence your decision?..... 393

(XXXI) From a histological standpoint, is the removal of a tooth pulp and the sealing of the canal a feasible operation? If not, why not? If so, under what conditions? .................... 393 
(XXXII) Do you believe in ionization? Why? To what extent?......... 393

(XXXIII) In case a vital pulp has been removed, and a radiogram shows that the end of the root was reached and a slight apical injury probably resulted, due to the severing of the pulp tissue, do you feel that the result of the operation will be influenced in any way by the presence of mouth infection, pyorrhea alveolaris, or apical abscesses? What do you do in such cases?

(XXXIV) In the case of a patient who fractures the two upper central incisors, what is your procedure (a) when the pulps are exposed; (b) when they are not exposed but you fear they will die? To what extent does the patient's age influence your decision?............ 393

Questions in the third part (pp. 225-298 of this volume):

(XXXV) In case the dentine of a non-vital tooth is not infected, and is surrounded by or encased in living cementum, which in turn is surrounded and nourished by a normal vital periodontal membrane, would you consider such a tooth a foreign body? Will it become infected, and will nature try to throw it off?.. 394

(XXXVI) Do you believe in treating diseased apical tissue through the canal, or do you believe in surgical removal of such tissue? .. 394

(XXXVII) Do you consider that a diseased tooth with infected dentine is more liable to have a deleterious effect on the surrounding tissues, even though the canal was cleansed of the infected pulp, than would be the case if the symptoms of inflammation of the tooth pulp were detected early and the pulp removed before it had become infected?.................... 394

(XXXVIII) To what extent do you consider $x$-ray evidence final in determining the occurrence, extent, and nature of apical pathological conditions? ............................... 394

(XXXIX) In orthodontic treatment, what causes pulp death during the process of moving the teeth and how can that cause or causes be eliminated? What do you do or advise when pulp death occurs for this reason?......................... 394

(XI) Do you think one should differentiate between teeth that contain infected pulps and teeth: rom which healthy pulps have been removed, in determining whether or not non-vital teeth may be retained?.

(XLI) (a) In correcting a case of malocclusion, do you first make a complete $x$-ray examination and vitality test of all the teeth in order to locate the unerupted or non-vital teeth? (b) If you make an examination, partial or complete, what do you do with the non-vital teeth present? (c) Do you complete your realignment with them in place or do you advise their early extraction? (d) What do you advise in case the third molars are impacted? (e) What do you think of the alveolus surrounding non-vital teeth as compared with that surrounding vital teeth? .................................... 
(XLII) In your opinion, is dentine infected by preparing cavities for fillings and then allowing some time to elapse before placing the filling, no precaution being taken to seal the cavity in the interim and to prevent admission to it of saliva?........ 395

(XLIII) May a non-vital tooth be free from infection?............ 395

(XLIV) May a vital tooth have an infected periodontal membrane (aside from that of a pyorrheal condition)? . . . . . . . . . . . 395

(XIV) Does an infection at a root end come (a) from the tooth itself (b) through the canal (c) by the presence of infected dentine; or (d) is it carried there from the blood stream independently of infection in the canal? In case you consider that an apical infection comes from the tooth itself or the canal, how do you account for (e) the presence of infection in canals and at apices that have undamaged natural crowns? (f) If you consider that such an infection comes from the blood stream, why is it that, in a given mouth, some non-vital teeth are not infected and others, adjacent, may be badly infected?..... 396

(XLVI) Do you believe that the presence of silicate fillings causes pulp death? If so, how do you prevent such action?......... 396

(XIVII) If you take the stand that all pulpless teeth, regardless of radiographic findings and regardless of the patient's health (good or poor), should be extracted, do you put any age limit on such procedure? Why?............................. 396

(XIVIII) Do you consider that the manner of handling hospital cases, relative to problems of pulpless teeth, should govern the management of patients in private practicei . . . . . . . 396

(XLIX) What do you think of the extraction of pulpless teeth that radiographically show no infection, in patients in good health?... 396

(L) How do you view the construction of extensive restorations that call for the use of pulpless teeth as abutments? .......... 396

(LI) What is your opinion on the use of large stationary bridges cemented to vital-tooth abutments? ................ 397

(LII) In case you had a pulpless tooth and a vital tooth proximating, with the pulpless tooth apparently free from infection and with a space adjacent to the pulpless tooth due to loss of teeth: Would you remove the pulpless tooth and carry the bridge to the vital tooth, or not?

III. Reference to the questions raised by correspondents in response to the general invitation, to them, to "suggest any other questions you think should be answered." ......................................... 397

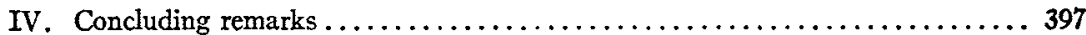




\section{INTRODUCTION}

The author presents, in the succeeding section, under a copy of each question in the questionnaire, a general summary of conclusions from the facts and opinions respectively stated by the correspondents, and published successively in the first three parts of this compilation.

The questions in the questionnaire are repeated in detail for the convenience of the reader, not only in the table of contents herewith but also in the text of this concluding part of the study.

II. COPIES OF THE QUESTIONS IN THE QUESTIONNAIRE; WITH THE AUTHOR'S SUMMARY, UNDER EACH, OF HIS CONCLUSIONS FROM

THE FACTS AND OPINIONS RESPECTIVELY STATED BY THE CORRESPONDEN'S

I. In the treatment of vital deciduous teeth, where the decay is so extensive that its entire removal would mean the exposure of the pulp, what do you advise and what is your procedure? Also, how do you proceed where the pulp is already exposed?

The consensus of opinion seems to be that every effort should be made to save the vitality of the pulp. Furthermore, there seems to be unanimity of feeling against exposure of the pulp in removing the involved dentin. Various methods are suggested for sterilization of the layer of dentin that has been left overlying the pulp. The inference seems to be that, if the pulp can be kept free from exposure, the chance of saving it is greatly enhanced.

II. In the treatment of deciduous teeth where the pulps have become non-vital and infected, what is your procedure and what do you advise?

While a certain percentage seem to favor extraction as the sole treatment for this condition, a majority make an effort, apparently, to differentiate between teeth to be saved by opening into, irrigating, and sealing root canals, and teeth that should be extracted. There is a noticeable absence of opinion that all such teeth should be saved. The use of the silver-reduction method appears to be the favorite where they are saved. There seems to be a common opinion that, if near the time for their exfoliation, the teeth involved should be removed. 
III. In case the root ends of permanent teeth are not completely formed and the pulp has decomposed, what is your procedure and what do you advise?

Opinions seem to be about equally divided as to whether such teeth should be extracted at the beginning or an attempt made to save them. A small number advocate opening through the buccal or labial alveolar process and sealing the canal at the apex. There seems to be a general inclination to regard the saving of such teeth as questionable procedure; and there is a marked tendency to "keep an eye on them," if they are left in the jaw.

IV. In case the root ends of permanent teeth are not completely formed, and the pulp is still vital but exposed, as a result of decay or trauma, what is your procedure and what do you advise?

A great majority favor efforts to save the pulp, even (as in the case of no. 39) where the pulp has become infected. A few favor removal of the pulp or extraction. Attention should be drawn to reference, by some, to the desirability of leaving the apical pulp tissue undisturbed, in the event of removal of the pulp.

V. In case the vital pulp is covered with a thin layer of decalcified dentine, what is your procedure and what do you advise?

Opinions are almost unanimous that every effort should be made to save the vitality of the pulp. Opinions differ greatly as to whether a layer of decalcified dentin should be left overlying the pulp or whether the pulp should be exposed. A majority seem to favor leaving a little dentin in the bottom of the cavity as a covering for the pulp. Use of the silver-reduction method is freely mentioned.

VI. At what age do you consider it good practice to begin bridgework that necessitates cutting into vital teeth? Do you consider that the location in the mouth is a governing factor?

The age is set all the way from sixteen years to middle life. However, there seems to be a general opinion that complete development of the roots should precede the use of such work. Special attention is drawn to the number who advise some form of restoration that does not necessitate cutting into sound enamel. The location of bridgework in the mouth is seen from two points of view; some stating that it is a governing factor, others that it is not. 
VII. When permanent teeth of young patients are extracted on account of apical infection, do you retain the spaces with orthodontic appliances, use bridgework, or pay no attention to the spaces created by the extractions?

A majority favor retaining the spaces. Special mention is made of removable appliances.

VIII. Where deciduous teeth are extracted, do you, under any circumstances, advise retention of the spaces with orthodontic appliances, for the eruption of the permanent teeth?

Opinions agree, in the main, that the spaces so created should be maintained, more especially if some time will elapse before eruption of the permanent teeth. A few are opposed to such a procedure.

IX. What were the conditions that caused the death or removal of so so many pulps in the past? To what extent are these conditions now under 'control and pulp removal unnecessary?

The responsibility is divided among physicians, dentists, and patients. Physicians have failed to appreciate the significance of dental caries and have neglected to advise patients to have carious teeth attended to. Patients fail to seek relief early enough, chiefly through fear of pain, which causes them to delay until carious cavities develop to the point where the pulp is involved. Also, many patients tolerate conditions of uncleanliness in their mouths that are conducive to development of caries. Dentists have been unaware of the results that frequently follow removal of the pulps of teeth, have used types of restorations necessitating pulp removal, and have failed to master the technic of preserving the vitality of pulps in teeth with large cavities. Relief can be assured (a) by educating the public, both adults and children, in the importance of clean, healthy mouths, free from dental caries; (b) when dentists perform dental operations without causing so much pain, use the newer types of dental restorations that do not require removal of pulps of teeth, and learn how to maintain pulp vitality in teeth with deep cavities; and (c) through the development of the specialty of pediadontia. The importance of deep study of each mouth, not only of individual teeth, is also mentioned. Great stress is laid on the practice of preventive dentistry as an important factor in keeping caries from progressing to a degree that requires removal of the pulp.

$\mathrm{X}$. What are the causes of pulp death over which we still have no control? 
Opinions are almost unanimous that trauma from accidental injury will continue to be one of the chief causes of pulp removal. Other causes over which we, as dentists, are not supposed to exercise control, are, neglect on the part of the patient, formation of deposits in the pulp chamber and canals, certain bad dental operations, and systemic conditions, including metastatic infection.

XI. In a case where decay is in actual contact with a vital pulp, do you advocate (a) removal of all decay and attempted pulp capping; or (b) removal of the decay followed by pulp removal, (c) leaving just enough decalcified dentine to prevent actual exposure and giving the case a treatment? (c) What does such treatment consist of? How long have you practised it? Do you consider it a good procedure to follow?

This question was asked because the author feels that much root-canal work has been due to the habit, of patients, of allowing large cavities to develop before seeking dental service, chiefly through fear of pain. The pulps in such cases have been removed.

While a number favor removal of the pulps in such cases, yet there is a noticeable tendency to save the pulps. The method of removing all dentin, partially or completely involved, seems to be giving way, in the opinions of many, to the plan of leaving even a thin layer of dentin over the pulp, on which is placed some material that will sterilize it. The results of this method of handling such cases, in the minds of many who use it, have been so gratifying that the attention of the reader is specially directed to it.

XII. How early do you begin oral prophylaxis for your patients?

The importance of early prophylaxis is shown by the unanimity of opinion to this effect. The prevailing idea is to start the supervision of the mouth as soon as possible.

XIII. Outline your plans for preventing decay and pulp death in the teeth of young patients.

The tendency to decay in children's teeth is recognized by all. The various valuable suggestions for the solution of the problem may be combined in one plan, as follows: Educate children and parents regarding mouth conditions in general. Teach a method for the adequate brushing of the teeth and use of dental floss; the latter, after each meal. Use an alkaline mouth wash. Areas showing decalcification of the enamel should be treated with silver nitrate. Occlusal 
fissures should be thoroughly cleaned with pics and broaches, and silver nitrate solution flowed into them; others should be cut into and filled; some should have cement forced into them. There should be regular prophylaxis at the office of the dentist. The value of the work of the dental hygienist should be emphasized.

XIV. Is it your habit to give the mouths of your patients prophylactic supervision during the time they are in your care? If so, how frequently do you see the patients for this work?

Opinions agree that there should be regular supervision of all patients. The time between recommended visits varies from two weeks to a year, according to conditions in the individual mouth. It is important that patients should be able to keep all surfaces of all teeth polished, and free from mucous plaques and food debris; and also to keep the gums free from signs of irritation.

$\mathrm{XV}$. In deciding why the radiograms of some non-vital teeth show areas indicative of apical disease, and some do not, and also why some persons with such teeth display symptoms suggestive of focal infection while others do not, is it probable that different factors contribute to the two conditions at the ends of the roots? (Be sure to mention whether or not you believe the development of apical infection is solely a matter of diminished resistance on the part of the patient.)

Many believe that diminished resistance on the part of the patient is a factor in such cases. Various other factors are mentioned, however, as contributing, if not responsible, agents for the condition.

XVI. In the case of a non-vital tooth showing apical infection, outline methods by which such infection can be removed and the tooth retained. Do you consider that your procedure gives permanently satisfactory results?

Opinions on this subject differ, although it is evident that all the correspondents aim to be as practical as possible. A majority attempt destruction of the apical infection through the root canal, and use various drugs for that purpose. The difficulty of doing this work on molars is mentioned by some. Quite a number favor a surgical method of getting rid of the infection-some by extraction and curettement, some by resection of the root tip. The uncertainty of the latter operation is mentioned by a number. One (no. 11) states that 90 per cent of such teeth have dead cementum, which can never again be united to vital tissue. 
XVII. Approximately what number of non-vital non-infected teeth would you advise leaving in any one mouth (a) in the absence of symptoms of focal infection; $(b)$ in the presence of symptoms of focal infection? Do you make any distinction, in this regard, as far as age of the patient is concerned?

The question is not sufficiently self-explanatory. It should convey the idea that, from a radiographic examination, we find no evidence of apical infection. The answers bring out quite clearly the fact that to extract pulpless teeth that show no apical disease, in patients who are in good health, is contrary to the practice of most of the correspondents. Where a patient is sick, there seems to be a marked tendency to have a consultation with the physician in charge before such teeth are removed. While there is uncertainty of opinion regarding the actual influence that pulpless teeth (non-infected so far as radiographic evidence is concerned) may have on a patient's health, there seems to be a strong feeling that an undesirable effect is not apt to occur.

XVIII. In a case showing symptoms of focal infection, to what extent would you go in trying to save a non-vital tooth that apparently had no apical infection, provided such a tooth was a valuable factor in the reconstruction of the mouth?

There is a certain range of opinions concerning the answer to this question. It runs from a purpose to save such teeth to a decision to extract them. Some recommend that such teeth be opened, cleansed, sterilized, and that the canals be sealed. Some open through buccal or labial plate and resect the root tip. Several advise elimination of all other foci before removing such teeth. The value of such teeth to the patient is referred to, on one hand; on the other, the probability of the good that removal of such teeth will do the patient is mentioned. The point of "putting it up to the patient" is also raised. Many express the opinion that, in serious cases, retention of such teeth is a somewhat "risky" procedure that is fraught, in some instances, with grave responsibilities. The answers show that no single rule for the handling of such cases can be adopted.

$\mathrm{XIX}$. In case a radiogram shows a tooth with a pulp chamber and portions of one or more roots only partly filled, how do you determine whether the unfilled apical portions of the roots contain vital pulp-tissue, 
or non-vital tissue, or no pulp-tissue at all-all three conditions being supposedly free from apical infection?

Most of the correspondents feel that there is no means, aside from exploring the canal with an instrument, of determining the vitality of apical pulp tissue. The efficacy of the electric current, in determining vitality or non-vitality of pulp tissue at the apex, is valued by some but considered of no value by others. Mention is made of close scrutiny of radiograms as an aid in determining effects on the pericemental membrane and the pericemental lamella (lamina dura) of the pulp tissue in the apex, in order to determine the probable condition of the pulp. Several show a desire to leave such teeth just as they are. Special mention is made of the fact that, after vitalpulp tissue was left in the apical portion, osteoblasts have closed the apical foramina.

$\mathrm{XX}$. In the reconstruction of a mouth, do you believe in removing non-vital teeth that do not show, radiographically, pathological conditions at the apices?

This question brings out the real opinion of the correspondents regarding pulpless teeth that radiographically show no apical disease. Many are very decided in the view that they would not remove such teeth under the circumstances; others would be influenced by the usefulness of such teeth in the reconstruction plan; others again prefer not to include pulpless teeth in the reconstruction of the mouth, as they consider them rather "uncertain."

XXI. Do you believe in root resection as a permanent operation? On which teeth? Do you feel that, in such an operation, the pulp-canal and dentin should receive special attention? Of what should such treatment consist?

There is an interesting and wide range of opinion in this relation. Some have no use at all for the operation. Probably the greater number, while having an apparent suspicion that it is hardly the correct thing (as they seem unable in some cases to reconcile themselves to the conditions after the operation), admit that they somewhat favor the operation. A point of particular interest is the fact that one correspondent favors it in certain cases, to avoid a break in an otherwise perfect arch. Those who favor the operation frequently mention it as being indicated in carefully selected cases. The need for care- 
ful attention to the root canal is extensively discussed. The indicated range for the operation varies from the centrals to the third molars.

XXII. Should a tooth that is abscessed be extracted during the active process of inflammation, or is such an operation as a routine procedure contraindicated?

A majority favor removal of such teeth, though some are opposed to it. Curetting after removal of the tooth is approved by some and opposed by others. Mention is frequently made of the need for careful post-operative dressings.

XXIII. For the extraction of a considerable number of teeth in a case of serious infection, how do you proceed? Do you remove a few at a time or all at one operation?

Most of the correspondents favor removal of a small number of teeth at a time. Among those who favor extensive removal of teeth, no. 23 refers to an interesting incident in his practice.

XXIV. Do you consider that unerupted and impacted teeth should be removed, regardless of their location, when they cannot be brought into their proper places in the arch?

While some favor removal of such teeth, on account of the trouble they may cause, a majority prefer to be governed by conditions affecting the individual case at the time a decision is made.

$\mathrm{XXV}$. Under what conditions, and in what locations, in the mouth, is it justifiable to remove a decomposed pulp and to fill the canal or canals? Give a list of such conditions.

The patient's physical condition and the accessibility of root canals appear to be governing factors in the minds of most of the correspondents. Many are disinclined to endeavor to save a tooth if there is any destruction of its apical tissue. Mention is made of the fact that although a portion of the pulp may be decomposed, the apical portion may remain vital. Certain replies indicate a desire to eliminate all teeth in this condition; others show a desire to have the patient share the responsibility of retaining them.

XXVI. Under what conditions, and in what locations in the mouth, is it justifiable to remove a vital pulp and to fill the root canals with an artificial substitute? Give a list of such conditions.

From the answers to this question it is apparent that most of the correspondents favor preservation of the vitality of the pulp as long 
as possible. Some consider that such conditions as exposures, pulp nodules, and secondary dentin, and the apparent early death of the pulp, justify its removal. An occasional correspondent would remove a pulp for purposes of reconstruction. Mention is made of contraindication in mouths in which there is much oral sepsis. The patient's health is also mentioned as a factor in determining whether a vital pulp should be removed.

XXVII. When a tooth, to be extracted, has an apical infection, should the infected region be curetted after the extraction, or should it be left undisturbed? Would your decision to curette be the same if the granuloma came out with the root?

A majority favor the curettement of infected sockets, but several express the opinion that it has been overdone. Opinions are divided as to whether or not a socket should be curetted when the granuloma adheres to the root of the extracted tooth.

XXVIII. Upon what basis do you decide what to do with the various teeth in a mouth that contains a great number of non-vital teeth; some showing apical involvement and some not; some with partially and others with completely filled roots; some with exposed vital pulps and some with dead pulps? (Go sufficiently into detail so that your answer may serve as a guide in general practice.)

There is almost a predominant opinion that the patient's health should govern the procedure in such cases. The importance of a carefully made oral examination, including full-mouth radiograms, study casts, vitality tests, and a careful clinical examination of the mouth, is referred to by several. Frequent mention is made of the desirability of removing all teeth that show apical infection. Reference is also made to the risk assumed in including questionable teeth in large reconstructions. Most of the answers show a desire to handle each tooth and each case with individual consideration. There is apparently no agreement on a plan of treatment for all cases of this type.

XXIX. In the case of a maxillary-sinus infection that did not respond to treatment, what disposition would you make of any non-vital molars or bicuspids on the side affected?

A majority favor removal of such teeth. 
$\mathrm{XXX}$. In the reconstruction of a mouth, would you advise the removal of one or more vital pulps, if that procedure enabled you to use a more satisfactory type of bridge than would be possible if such pulps were not removed? If you ever advise such a procedure, does the patient's age and condition influence your decision?

Opinions on this subject are divided, with those opposed to such a procedure in a majority. There is quite a range of opinion as to the effect of age on such a procedure.

XXXI. From a histological standpoint, is the removal of a tooth pulp and the sealing of the canal a feasible operation? If not, why not? If so, under what conditions?

Most of the correspondents feel that from a practical standpoint the operation may be satisfactory, but a much smaller number feel satisfied with it from a histological point of view. Of the latter, six give a very interesting explanation of what takes place in the apical portion of a root when a small portion of vital pulp is left uninjured. Several hold a somewhat different view on this particular point.

XXXII. Do you believe in ionization? Why? To what extent?

While some feel that "ionization" is a real aid in their practice, a majority are not very enthusiastic over it.

XXXIII. In case a vital pulp has been removed, and a radiogram shows that the end of the root was reached and a slight apical injury probably resulted, due to the severing of the pulp tissue, do you feel that the result of the operation will be influenced in any way by the presence of mouth infection, pyorrhea alveolaris, or apical abscesses? What do you do in such cases?

While a variety of opinions are expressed on this question, many believe, on general principles, that mouth infection, gingival and apical, should be eradicated before such an operation is undertaken.

XXXIV. In the case of a patient who fractures the two upper central incisors, what is your procedure (a) when the pulps are exposed; (b) when they are not exposed but you fear they will die? To what extent does the patient's age influence your decision?

The desirability of saving the pulp, if at all possible, is emphasized by most of the correspondents. However, when there is a condition as in (a), most of the replies indicate that the pulps should be removed and the roots filled. There are some exceptions to this, however. 
In case of (b), need for the most careful supervision of the case is emphasized, particularly in younger patients.

XXXV. In case the dentin of a non-vital tooth is not infected, and is surrounded by or encased in living cementum, which in turn is surrounded and nourished by a normal vital periodontal membrane, would you consider such a tooth a foreign body? Will it become infected, and will nature try to throw it off?

Such teeth, in the opinion of practically all the correspondents, are not foreign bodies and nature will not attempt to throw them off. (See no. 18 for a slightly different view.)

XXXVI. Do you believe in treating diseased apical tissue through the canal, or do you believe in surgical removal of such tissue?

Opinions are divided on this question, but many feel that, when such conditions are treated through the canal, it should be in the simpler cases only. Ionization is referred to, but, for extensive apical involvement, the majority favor surgery. The patient's physical condition as a factor is mentioned.

XXXVII. Do you consider that a diseased tooth with infected dentin is more liable to have a deleterious effect on the surrounding tissues, even though the canal was cleansed of the infected pulp, than would be the case if the symptoms of inflammation of the tooth pulp were detected early and the pulp removed before it had become infected?

There is very little difference of opinion on this subject. The tooth with infected pulp removed "runs a poor second."

XXXVIII. To what extent do you consider $x$-ray evidence final in determining the occurrence, extent, and nature of apical pathological conditions?

The answers to this question are practically unanimous. They show quite clearly that while the radiogram is an extremely valuable aid in diagnosis, it is still to be considered solely as an aid and cannot be accepted as always showing indisputable evidence.

XXXIX. In orthodontic treatment, what causes pulp death during the process of moving the teeth and how can that cause or causes be eliminated? What do you do or advise when pulp death occurs for this reason?

Most of the correspondents concur in the opinion that pulp death in such cases occurs as a result of either too rapid movement of a tooth, or excessive stress upon a tooth in the closure of the jaws due 
to change in position of the tooth. Either of these conditions causing an injury to the apical tissues, the circulation to the tooth is interfered with and pulp death results. Most of the correspondents recommend the saving of such a tooth, but the question of the development of the root is raised by some and its extraction advised.

$\mathrm{XL}$. Do you think one should differentiate between teeth that contain infected pulps and teeth from which healthy pulps have been removed, in determining whether or not non-vital teeth may be retained?

Some feel that no distinction should be made, but a majority favor the tooth from which a healthy pulp had been removed. One correspondent raises the point that, in a sick patient, no distinction whatever should be made and that the pulpless teeth should be removed.

XII. (a) In correcting a case of malocclusion, do you first make a complete $x$-ray examination and vitality test of all the teeth in order to locate the unerupted or non-vital teeth? (b) If you make an examination, partial or complete, what do you do with the non-vital teeth present? $(c)$ Do you complete your realignment with them in place or do you advise their early extraction? (d) What do you advise in case the third molars are impacted? (e) What do you think of the alveolus surrounding nonvital teeth as compared with that surrounding vital teeth?

Opinions on the various points raised in this question vary considerably. A review of each answer is necessary.

XLII. In your opinion, is dentin infected by preparing cavities for fillings and then allowing some time to elapse before placing the filling, no precaution being taken to seal the cavity in the interim and to prevent admission to it of saliva?

While a few feel that dentin under such conditions is not infected, a majority believe that it is and that precautions should be taken to protect it.

XLIII. May a non-vital tooth be free from infection?

There is a division of opinions on this subject, but the view predominates that such a tooth may be free from infection.

XLIV. May a vital tooth have an infected periodontal membrane (aside from that of a pyorrheal condition)?

A majority believe that such a condition can easily exist. There is an interesting array of probable causes for such a condition. 
$\mathrm{XIV}$. Does an infection at a root end come (a) from the tooth itself (b) through the canal (c) by the presence of infected dentine; or $(d)$ is it carried there from the blood stream independently of infection in the canal? In case you consider that an apical infection comes from the tooth itself or the canal, how do you account for (e) the presence of infection in canals and at apices that have undamaged natural crowns? (f) If you consider that such an infection comes from the blood stream, why is it that, in a given mouth, some non-vital teeth are not infected and others, adjacent, may be badly infected?

Most of the correspondents believe that root-end infection comes through the canal, but several express the belief that it may arrive by way of the blood stream or from the gingival crevice.

XLVI. Do you believe that the presence of silicate fillings causes pulp death? If so, how do you prevent such action?

There is a decided difference of opinion as to whether or not silicate fillings cause pulp death. A great many favor the use of some kind of a base or cavity lining for the purpose of protecting the pulp.

XIVII. If you take the stand that all pulpless teeth, regardless of radiographic findings and regardless of the patient's health (good or poor), should be extracted, do you put any age limit on such procedure? Why?

Opinions are practically unanimous in opposing such a stand.

XIVIII. Do you consider that the manner of handling hospital cases, relative to problems of pulpless teeth, should govern the management of patients in private practice?

There seems to have been a little misunderstanding of the point in this question. Nevertheless, there appears to be a difference of opinion whether a patient in private practice should have his pulpless teeth handled as they generally are in a hospital.

XLIX. What do you think of the extraction of fulpless teeth, that radiographically show no infection, in patients in good health?

Opinions are practically unanimous in condemning such practice.

L. How do you view the construction of extensive restorations that call for the use of pulpless teeth as abutments?

Some of the answers indicate willingness to utilize such teeth as abutments in reconstructive operations, providing radiograms show no apical-tissue destruction. A majority, however, are opposed to such practice. The answers to this question show some very interesting views on different types of bridgework. 
LI. What is your opinion on the use of large stationary bridges cemented to vital-tooth abutments?

Interesting differences of opinion were expressed. A majority are opposed to the use of this type of bridge.

LII. In case you had a pulpless tooth and a vital tooth proximating, with the pulpless tooth apparently free from infection and with a space adjacent to the pulpless tooth due to loss of teeth: Would you remove the pulpless tooth and carry the bridge to the vital tooth, or not?

As a general rule, the correspondents show a desire to use the pulpless tooth. The patient's health and the quality of the root-canal operation are mentioned as factors of influence on the decision to save or extract.

III. REFERENCE TO THE QUESTIONS RAISED BY CORRESPONDENTS IN RESPONSE TO THE GENERAL INVITATION, TO THEM, TO "SUGGEST ANY OTHER QUESTYONS YOU THINK SHOUID BE ANSWERED."

Answers to the questions raised by the correspondents (page 295) will not be included in this work. It had been the author's expectation to publish a large number of answers to each question. This purpose required submission of each question to each correspondent. Execution of this plan would delay publication of the work to such an extent, however, judging from the experience in obtaining answers to the first set of questions, that the inevitable postponement would be unfair to those who have unselfishly given their time to the effort to make this study a success.

\section{CONCLUDING REMARKS}

In conclusion, the author expresses the hope that this compilation will very materially help to solve some of the problems that have been disturbing the minds of the members of the dental profession.

933 Metropolition Bank Building. 